\title{
Repeatability of two semi-automatic artificial intelligence approaches for tumor segmentation in PET
}

\author{
Elisabeth Pfaehler ${ }^{1 *}$ (D) Liesbet Mesotten ${ }^{2,3}$, Gem Kramer ${ }^{4}$, Michiel Thomeer ${ }^{2,5}$, Karolien Vanhove ${ }^{2,6}$, \\ Johan de Jong ${ }^{1}$, Peter Adriaensens ${ }^{7}$, Otto S. Hoekstra ${ }^{4}$ and Ronald Boellaard ${ }^{1,4}$
}

\begin{abstract}
Background: Positron emission tomography (PET) is routinely used for cancer staging and treatment follow-up. Metabolic active tumor volume (MATV) as well as total MATV (TMATV-including primary tumor, lymph nodes and metastasis) and/or total lesion glycolysis derived from PET images have been identified as prognostic factor or for the evaluation of treatment efficacy in cancer patients. To this end, a segmentation approach with high precision and repeatability is important. However, the implementation of a repeatable and accurate segmentation algorithm remains an ongoing challenge.
\end{abstract}

Methods: In this study, we compare two semi-automatic artificial intelligence (Al)-based segmentation methods with conventional semi-automatic segmentation approaches in terms of repeatability. One segmentation approach is based on a textural feature (TF) segmentation approach designed for accurate and repeatable segmentation of primary tumors and metastasis. Moreover, a convolutional neural network (CNN) is trained. The algorithms are trained, validated and tested using a lung cancer PET dataset. The segmentation accuracy of both segmentation approaches is compared using the Jaccard coefficient (JC). Additionally, the approaches are externally tested on a fully independent test-retest dataset. The repeatability of the methods is compared with those of two majority vote (MV2, MV3) approaches, $41 \% S U V_{\text {MAX, }}$ and a SUV $>4$ segmentation (SUV4). Repeatability is assessed with test-retest coefficients (TRT\%) and intraclass correlation coefficient (ICC). An ICC $>0.9$ was regarded as representing excellent repeatability.

Results: The accuracy of the segmentations with the reference segmentation was good (JC median TF: 0.7, CNN: 0.73). Both segmentation approaches outperformed most other conventional segmentation methods in terms of

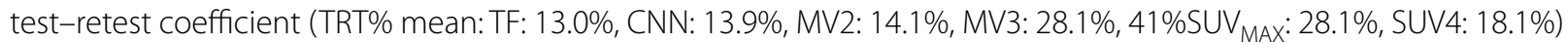

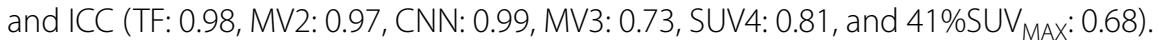

Conclusion: The semi-automatic Al-based segmentation approaches used in this study provided better repeatability than conventional segmentation approaches. Moreover, both algorithms lead to accurate segmentations for both primary tumors as well as metastasis and are therefore good candidates for PET tumor segmentation.

Keywords: Repeatability, Textural segmentation, Convolutional neural network, Tumor segmentation PET

*Correspondence: e.a.g.pfaehler@umcg.nl

${ }^{1}$ Department of Nuclear Medicine and Molecular Imaging, Medical Imaging Center, University of Groningen, University Medical Center Groningen, Groningen, The Netherlands

Full list of author information is available at the end of the article

\section{Introduction}

Positron emission tomography in combination with computed tomography (PET/CT) using the tracer fluorodeoxyglucose (FDG) is an important imaging modality for cancer diagnosis, tumor staging, prognosis or treatment follow-up $[1,2]$. The volume of the segmented tumor in the PET image, also known as metabolic active tumor 
volume (MATV) as well as the total MATV (TMATVincluding metastasis and lymph nodes), is one important metric for the evaluation of therapy response [3]. Observed differences in MATV/TMATV should reflect actual tumor volume differences and not segmentation errors. Therefore, a repeatable segmentation is of utmost importance. Hereby, a repeatable segmentation refers to a segmentation algorithm leading to comparable results when applied on two consecutive PET/CT images of the same patient under the same physiological conditions. The implementation of a repeatable segmentation algorithm is not trivial due to the challenges associated with PET images. Among them are factors regarding the image quality, e.g., the low signal-to-noise ratio, low spatial resolution and partial volume effects. Especially for smaller lesions, the partial volume effect can reduce the apparent tumor uptake, making the lesion difficult to detect and segment.

Up to now, a manual segmentation by an expert or (if available) a consensus segmentation of several experts are considered as gold standard. However, manual segmentations have several drawbacks, e.g., they are timeconsuming, non-reproducible and come with a high inter-observer variability [4-6]. A recent study also demonstrated that even the consensus of several observers results in a low repeatability compared to automated segmentations [7].

To overcome the limitations of manual segmentations and to increase repeatability, a large number of (semi-) automatic segmentation methods have been developed. The most basic and frequently used ones are thresholding algorithms defining voxels with an intensity value above a certain threshold as part of the tumor [8]. Also adaptive and iterative algorithms are available which adapt the threshold according to the actual image characteristics [9]. However, the performance of all these thresholding approaches depends on the scanner type, reconstruction algorithm, as well as image noise and have therefore limitations [10].

Therefore, more robust segmentation algorithms have been developed aiming to improve segmentation accuracy and repeatability. These include methods using the statistical properties of the image as well as learningbased methods [11, 12]. Nevertheless, most of these approaches have only been tested on limited datasets and are not widely available. Therefore, (semi-) automated segmentation methods used in the clinic are still mainly simple threshold-based approaches.

Due to the mentioned limitations of available segmentation algorithms, there is the need for new, more robust segmentation approaches. Artificial intelligence (AI)-based segmentations such as convolutional neural networks $(\mathrm{CNN})$ have shown very promising results for various segmentation tasks [13] and yield great promise for the segmentation of tumors in PET images. However, only a few studies use AI-based segmentation approaches for metabolic active tumor segmentation in PET images. Moreover, most studies combine the information of PET and $\mathrm{CT}$ images in order to get reliable segmentation results [14] or use some post-processing for an improvement of CNN segmentations [15]. Algorithms classifying each voxel as tumor or non-tumor using textural features of voxel neighborhoods have been used for the segmentation of e.g., lung carcinoma or head-and-neck cancer [16-18]. All of these studies combine the information of PET and CT images. In many cases, the PET/CT is performed with a low-dose CT, the latter does not have an optimal image quality for segmentation purposes. Therefore, it is of interest to develop AI-based PET segmentations that rely on PET information only. Additionally, in previous papers, segmentation approaches were applied on primary tumors only, while for the calculation of TMATV, an accurate and repeatable segmentation of metastasis and lymph nodes is important. This task is especially challenging due to the small size of metastasis, different tumor-to-background ratios and different locations of the metastasis in the body.

While several studies already reported the segmentation accuracy of AI-based segmentation algorithm, to the best of our knowledge, no study reported yet the repeatability of those algorithms. In this study, we investigate the repeatability of two AI approaches especially built to segment both primary tumors and metastasis accurately and repeatably. We focus on the segmentation task and do not consider lesion detection. This study includes a textural feature-based segmentation approach as well as a 3D CNN. All algorithms are trained, validated and tested on a dataset of Non-Small-Cell-Lung-Cancer (NSCLC) patients. Moreover, the algorithms are applied to a fully independent test-retest dataset of ten NSCLC patients scanned on two consecutive days. The repeatabilities of the AI segmentation approaches are compared with those of conventional segmentation algorithms used in the clinic.

\section{Materials and methods \\ Datasets}

The study was registered at clinical trials.gov (NCT02024113) and was approved by the Medical Ethics Review Committee of the Amsterdam UMC and registered in the Dutch trial register (trialregister.nl, NTR3508). All patients gave informed consent for study participation and use of their data for (retrospective) scientific research. Two datasets acquired at two institutions were included in this study with both datasets following the recommendations of the 
EARL accreditation program $[19,20]$. All images were converted to Standardized Uptake Value (SUV) units before the segmentation process started in order to normalize the images for differences in injected tracer dose and patient weight. This paper focuses on the segmentation process and not on lesion detection. Therefore, before the start of the segmentation process, a large bounding box was drawn around every lesion including also a large number of non-tumor voxels as illustrated in Fig. 1. The bounding box was drawn randomly such that the tumor was not always appearing in the middle but on different locations in the box. This step was performed in order to avoid that the CNN remembers the location of the object instead of other, more important characteristics. As a $\mathrm{CNN}$ requires that all images have the same size, each bounding box had a size of $64 \times 64 \times 64$.

\section{Training and testing dataset}

For training, validating and testing the segmentation approaches, 96 images of patients with NSCLC Stage III-IV were included. Patients fasted at least six hours before scan start and were scanned $60 \mathrm{~min}$ after tracer injection. All images were acquired on a Gemini TF Big Bore (Philips Healthcare, Cleveland, OH, USA). For attenuation correction, a low dose CT was performed. All images were reconstructed to a voxel size of $4 \times 4 \times 4 \mathrm{~mm}$ using the vendor provided BLOB-OSTOF algorithm. More details about the patient cohort can be found in previous studies [21]. Fivefold crossvalidation was performed whereby randomly $70 \%$ of the images were used for training, $10 \%$ for validation and $20 \%$ for independent testing.

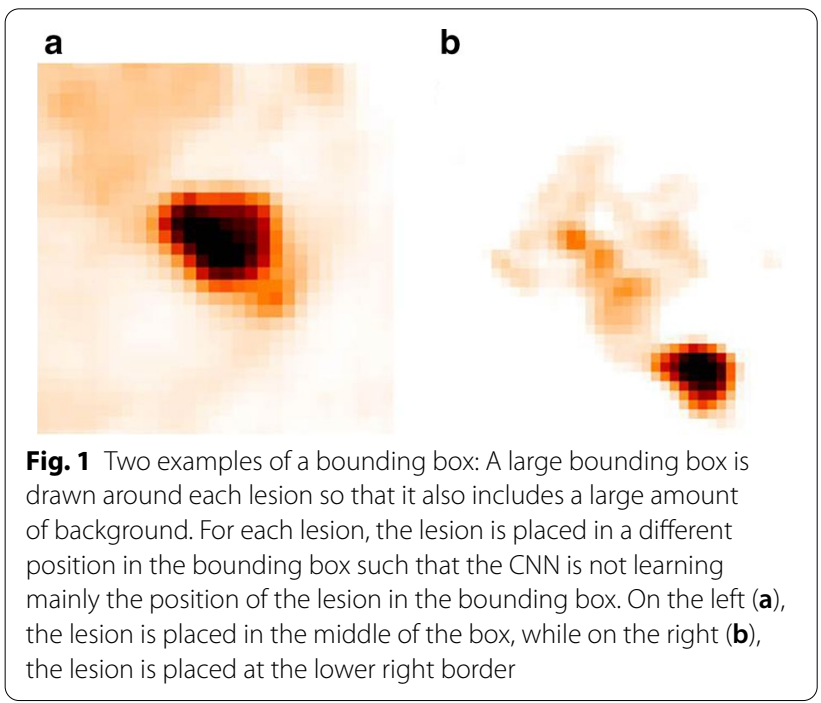

\section{Test-retest dataset}

For a fully independent test-retest evaluation, ten PET/ CT scans of patients with Stage III and IV NSCLC were analyzed. These ten patients underwent two wholebody PET/CT scans on two consecutive days. Images were acquired on a Gemini TF PET/CT scanner (Philips Healthcare, Cleveland, OH, USA) at a different institution (Amsterdam University Medical Center). Patient fasting time, time between tracer injection and scan start, as well as reconstruction algorithm and voxel size were the same as in the previous described dataset. A total of 28 lesions were included in the analysis.

\section{Reference segmentations}

The reference segmentations used for training, validating and testing the algorithm were obtained by applying an automatic segmentation which identified all voxels with a SUV above 2.5 as tumor (here after SUV2.5). The segmentations were manually adjusted by an expert medical physicist (RB) with more than twenty years of experience in PET tumor segmentation. This approach was chosen as it has been demonstrated that the manual adaption of a (semi-) automatic algorithm is more robust than a pure manual segmentation [22].

\section{Segmentation algorithm}

All segmentation algorithms were implemented in Python 3.6 using the libraries keras and scikit-learn.

\section{Convolutional neural network (CNN)}

A 3D CNN following the U-Net architecture proposed by Ronneberger et al. [23] was implemented with the keras library. U-net is one of the most famous and most frequently used $\mathrm{CNN}$ architectures for biomedical image segmentation, and it was especially designed for scenarios where only a small number of training examples are available. An illustration of the used architecture is displayed in Fig. 2. An U-Net consists of an encoding and decoding part. In the encoding part, the images are subsequently down-sampled while the number of features is increased. In the decoding part, the images are up-sampled while the number of features decreases. In both parts, three layers consisting of one convolutional block (=two convolutional layers with a kernel size of 5 followed by a Rectified Linear Unit ( $R e L u$ ) layer), a max-pooling layer for down-sampling in the encoding or a convolutional up-sampling layer in the decoding part, a batch normalization layer to increase network convergence and a drop-out layer to avoid overfitting. Due to the relatively small dataset, the CNN was trained with 8 initial features in the first layer. The number of layers and initial features 


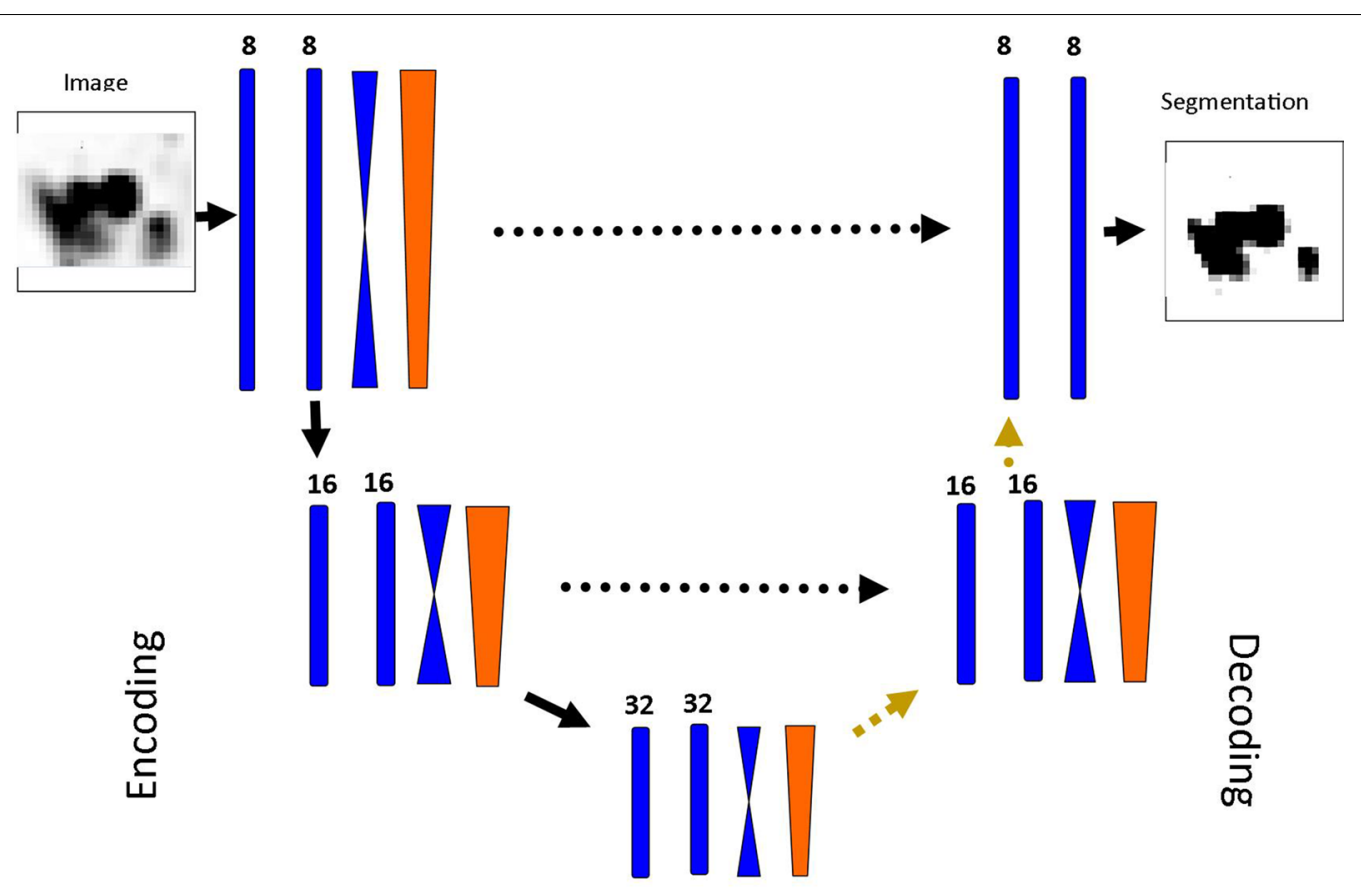

Conv, $5 \times 5$, ReLu

1 Max. Pooling 2D

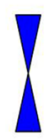

Batch normalization

Dropout

\section{- Up conv.}

Fig. 2 CNN architecture: In the encoding path, the images are subsequently downsampled while the number of features increases. In the decoding path, the images are upsampled while the number of features decreases. Encoding and decoding path are connected with skip connections

were determined iteratively until the validation accuracy was optimal and at the same time comparable to the accuracy in the training set. The latter is important as a large difference in training and validation accuracy is a hint for overfitting. Details about training and validation accuracy for different number of initial features can be found in the Additional file 1: Tables S1 and S2. The CNN was trained for 1000 epochs with a batch size of 25. The learning rate was set to 0.001 and an Adamoptimizer was used for weight adaptation. The negative Dice-coefficient was used as loss function measuring the overlap of two segmentations. A Dice coefficient of 1 is reflecting a perfect overlap. A U-Net requires that all images have the same size.

In order to increase the amount of training data and to avoid over-fitting, data augmentation was performed. This included rotations within $-20^{\circ}$ to $20^{\circ}$, shifting in width and height direction within $20 \%$ of the side length, a rescaling of the images within $25 \%$, intensity stretching, as well as adding Gaussian noise to the image.
For training, testing and applying the $\mathrm{CNN}$, the dataset was divided into smaller $(\leq 12.8 \mathrm{ml})$ and bigger tumors. The threshold was chosen empirically, and it was found that this threshold led to the best performance. For each tumor size category, one separate $\mathrm{CNN}$ was trained. Splitting the dataset by lesion size was performed as this led to more accurate and repeatable segmentations (illustrated in Additional file 1: Section 4). In order to train the two separate networks, lesions were selected using the volume of the ground truth mask. Depending on this tumor size, the lesion was used for training the corresponding CNN. After training and testing the CNNs, the appropriate $\mathrm{CNN}$ for a specific lesion was selected based on an initial guess of the tumor size. The latter is obtained using a majority vote (MV) segmentation. This MV segmentation uses four standard threshold approaches as input (see explanation below and Additional file 1: Section 5). The MV segmentation was chosen for this task because it provided the most accurate segmentations when compared with manual segmentations in previous 
work [7], and it is easy to implement. This initial tumor MV segmentation was only performed to select the corresponding $\mathrm{CNN}$, i.e., to distinguish between smaller and bigger lesions.

\section{Textural feature segmentation (TF)}

In the TF segmentation approach, textural features of voxel neighborhoods were used for the voxel-wise segmentation of the tumor. For every view (axial, sagittal, coronal), a separate segmentation was performed, and the summed probability was used to generate the final segmentation. The workflow of the TF segmentation for one image view is illustrated in Fig. 3. As illustrated, every voxel was regarded as center of a scanning window. For each scanning window, statistical and textural features were calculated using the open-source software pyradiomics [24]. The feature space was then reduced by selecting the most important features for the segmentation task, which were identified by a random forest.

Next, a random forest classifier was trained to classify each voxel as tumor or non-tumor. The trained random forest was then applied to the testing dataset. The probability images of the three orientations are summed in order to obtain the final classification. A probability image contains information on the certainty of the classifier making the right decision. All voxels with a summed probability of more than 1.8 were included in the final tumor segmentation. A more detailed description of the algorithm can be found in Additional file 1 and in Pfaehler et al. [25].
To evaluate how well the AI-based segmentations were matching the reference segmentation, the overlap between the AI-based segmentations and those of the reference segmentation were analyzed using Jaccard Coefficients, as explained later.

\section{Conventional segmentation algorithm}

The repeatability of the AI-based segmentations was compared with two established segmentation algorithm:

- $41 \% \mathrm{SUV}_{\mathrm{MAX}}$ : all voxels with intensity values higher than $41 \%$ of the maximal SUV value $\left(\mathrm{SUV}_{\mathrm{MAX}}\right)$ are regarded as tumor

- SUV4: all voxels with a SUV higher than 4 are included in the segmentation

Moreover, two majority vote (MV) approaches combining four frequently used thresholding approaches were included in the comparison. Both MV approaches were previously found to be more repeatable than conventional approaches [7]. The underlying segmentation algorithms were the above described SUV4 and $41 \%$ SUVMAX method as well as a segmentation including all voxels with a SUV above 2.5 and a $50 \%$ of SUVmax threshold-based segmentation with background correction. The two MV segmentation methods include:

- MV2: the consensus of at least two of the 4 standard approaches

- MV3: the consensus of at least three of the 4 standard approaches

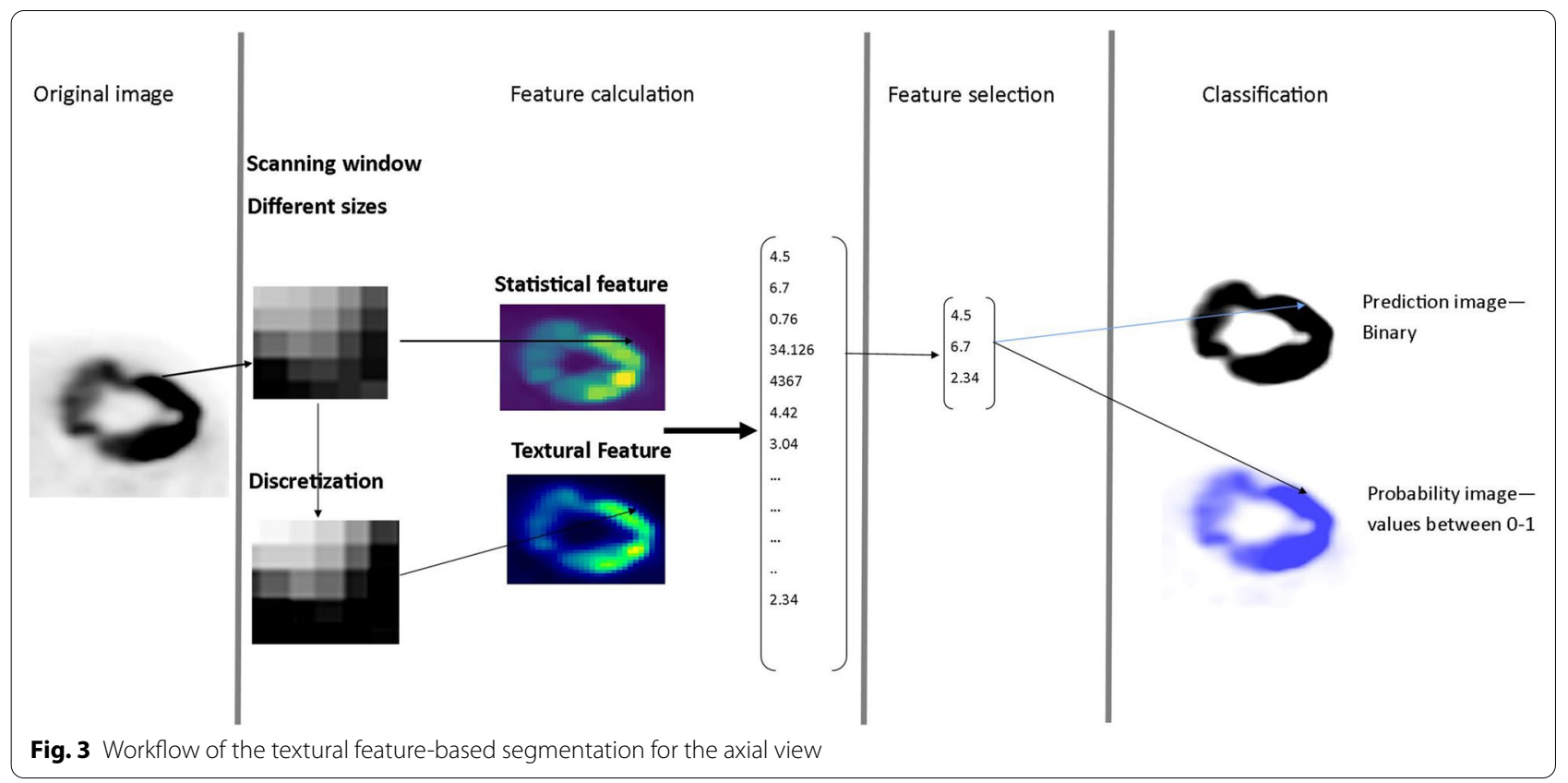




\section{Evaluation of segmentation algorithm}

For the evaluation of the segmentation algorithms, several metrics will be reported. Data analysis was performed in Python 3.6.2 using the packages numpy and scipy.

\section{Accordance of Al segmentation and reference segmentation}

In order to determine the accordance of the $\mathrm{AI}$ and reference segmentations, the Jaccard Coefficient (JC) was calculated. The JC is defined as the ratio between the intersection and the union of two labels and gives an indication about the overlap of the two labels:

$$
\mathrm{JC}=\frac{A \cap B}{A \cup B}
$$

A JC of 1 indicates perfect overlap, while a JC of 0 indicates that there is no overlap at all.

Furthermore, as the JC does not contain information about volume differences, the ratio between the volume of $\mathrm{AI}$ and reference segmentations were calculated: $\frac{M_{\text {MTV }}}{\text { MATVGM }_{\text {REF }}}$. A volume ratio above 1 indicates an over- and a volume ratio below 1 an under-estimation of the volume. A ratio of 1 represents perfect alignment. Finally, the distance of mass (barycenter distance) of the segmentations was calculated. Hereby, a barycenter distance close to 0 indicates perfect agreement.

\section{Repeatability evaluation}

The repeatability of the segmentation approaches was evaluated by comparing the differences of segmented volumes across days. For this purpose, the percentage testretest difference (\%TRT) was calculated:

$$
\mathrm{TRT} \%=\frac{\left|\operatorname{vol}_{\text {Day1 }}-\operatorname{vol}_{\text {Day2 }}\right|}{\left(\operatorname{vol}_{\text {Day1 }}+\operatorname{vol}_{\text {Day2 } 2}\right) / 2} * 100
$$

The \%TRT measures the proportional differences in segmented volume between the two consecutive scans. Moreover, the repeatability coefficient (RC) which is defined as $1.96 \times$ standard deviation (TRT\%) was calculated. Additionally, intraclass correlation coefficients (ICC) were calculated using a two-way mixed model with single measures checking for agreement. An ICC between 0.9 and 1 indicates excellent and an ICC between 0.75 and 0.9 indicates good repeatability [26]. If a lesion was completely missed by one segmentation approach, it was discarded from the analysis to analyze the same dataset for all segmentation approaches.

The accuracy metrics of the AI-based segmentations as well as the TRT\% of all approaches were compared using the Friedman test. The Friedman test is a non-parametric test, which does not assume a normal distribution of the data or independency of observations. It compares the rank of each data point instead of only comparing mean or median values. This means that if a segmentation algorithm provides consistently more accurate results, it will be ranked higher even if its mean or median are lower. As the Friedman test only contains information to show a significant difference in the data, a Nemenyi test was performed in order to assess which methods resulted in significant differences. P-values below 0.01 were considered as statistically significant. A Benjamini-Hochberg correction was applied in order to correct for multiple comparisons.

\section{Results}

\section{Accordance reference: Al-based segmentation}

Figure 4 displays the JC values between AI-based and reference segmentations for the cross-validation and test-retest dataset. The results of the separate folds are displayed in Additional file 1: Figure S8 and S9. In both cases, both approaches resulted in similar accuracies, which were not significantly different $(p>0.01)$. In the testing dataset, both approaches yielded good JC values (TF: median: $0.68,25$ th percentile: 0.46 , 75 th percentile: $0.83, \mathrm{CNN}$ : median: $0.7,25$ th percentile: 0.52 , 75 th percentile: 0.84 ) indicating a good accordance with the reference segmentations. Volume ratios and barycenter distances are listed in Table 1 . The CNN yields less underestimations and more overestimations of tumor volume [higher volume ratios (25th/75th percentile: $0.75 / 1.21)$ ]. While the TF approach resulted in more underestimations of tumor volume (25th/75th percentile: $0.68 / 1.08$ ). The barycentric distances of the TF approach were lower than the barycentric distances of the CNN. The corresponding values for the test-retest dataset can be found in Additional file 1: Table S3.

In general, the accuracy of the segmentations depended on the lesion size as illustrated in Fig. 5. Segmentations of bigger tumors resulted in better accuracy than segmentations of smaller lesions. For larger lesions, the $\mathrm{CNN}$ resulted in a median $\mathrm{JC}$ value of 0.74 , while the TF approach yielded a median JC of 0.82 . For smaller lesions, the $\mathrm{CNN}$ yielded a median JC value of 0.7 which was higher than the median of the TF approach (0.56). For larger lesions, the $\mathrm{CNN}$ had a median volume ratio of 0.92 (25th/75th percentile: $0.81 / 1.13)$. While for smaller lesions, the CNN resulted in a median volume ratio of 0.92 (25th/75th percentile: 0.73/1.18). These results indicate that the $\mathrm{CNN}$ resulted in a similar number of overestimations for small and large lesions. While the TF approach yielded in the majority of the cases volume ratios below 0.8 and therefore for smaller and larger lesions more underestimations. All JC values, volume 

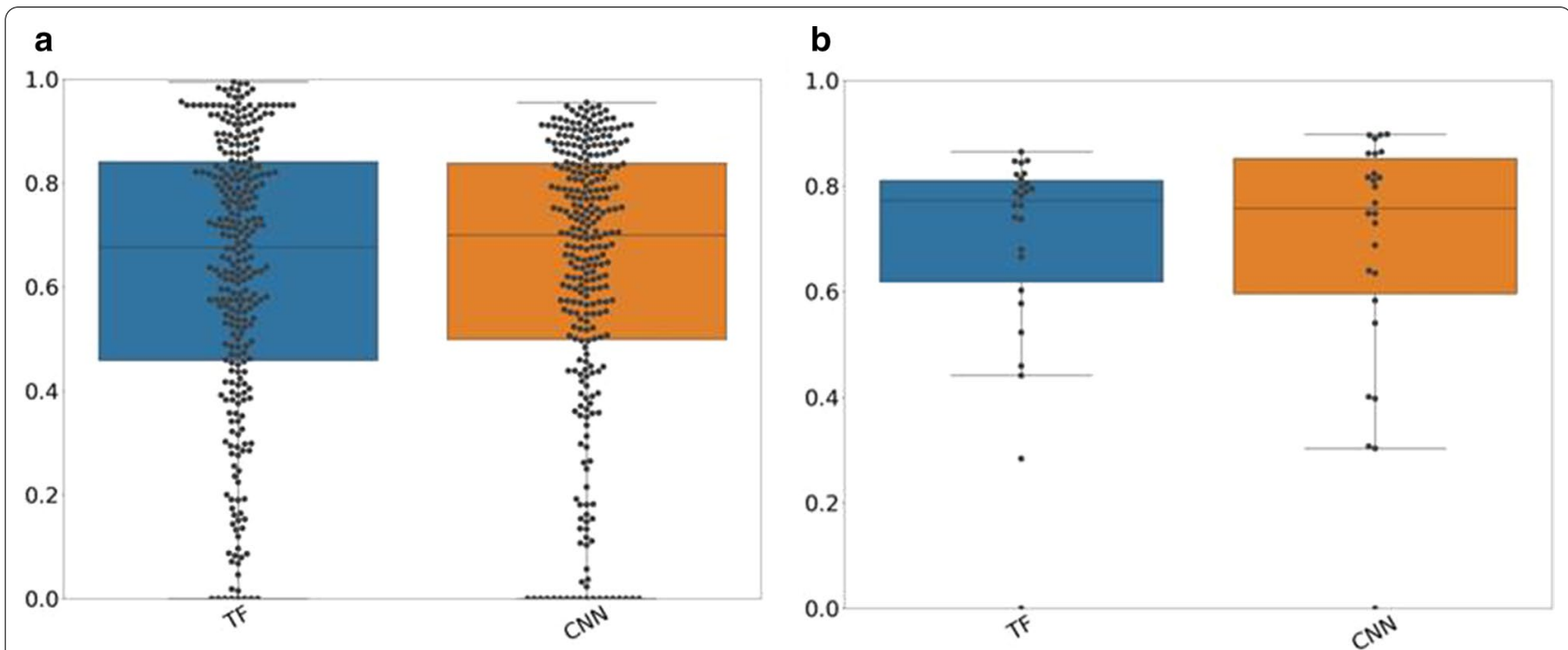

Fig. 4 Jaccard coefficient (JC) values for both datasets: JC values for the cross-validated dataset (left figure) and the test-retest dataset (right figure) for the Al-based segmentation algorithm included in the study

Table 1 Volume ratios and barycentric distances for TF and CNN

\begin{tabular}{lll}
\hline & $\begin{array}{l}\text { Volume ratio } \\
\text { Median (25th/75th } \\
\text { quartile) }\end{array}$ & $\begin{array}{l}\text { Barycentric distance } \\
\text { Median (25th/75th } \\
\text { quartile) }\end{array}$ \\
\hline TF & $0.70(0.59 / 0.79)$ & $0.68(0.42,1.66)$ \\
CNN & $0.99(0.83 / 1.34)$ & $0.81(0.36,2.1)$ \\
\hline
\end{tabular}

ratios and barycentric distances for smaller and larger lesions are listed in Table 2.

As displayed in Fig. 5, TF and CNN resulted in three cases with JC values around or below 0.4 for bigger lesions. In these cases, the tumors were located close to the heart, which was incorrectly included in the segmentation. Therefore, the tumor volume was highly overestimated. A similar effect was observed for smaller lesions: The CNN did not segment some of the smaller lesions while this was not the case for the TF-based approach. All lesions that were completely missed were located close to the kidneys, which was wrongly identified as tumor. The TF approach segmented the kidney regions but still included the tumors in the final segmentation.

\section{Repeatability}

Figure 6 displays the TRT\% for all segmentation algorithms. Two lesions were completely missed by the $\mathrm{CNN}$ and therefore discarded from the analysis.
CNN-based segmentations outperformed the other approaches regarding TRT\% with an absolute mean value of $13.9 \%$ and a standard deviation of $16 \%$. TF and MV2 segmentation yielded absolute mean values of $13.0 \%$ and $14.1 \%$ and standard deviations of $17 \%$ and 21\%, MV3, 41\%SUVMAX and SUV4 segmentations yielded mean values of $28.1 \%, 28.1 \%$ and $18.1 \%$, and standard deviations of $50 \%, 51 \%$ and $26 \%$. The corresponding repeatability coefficients can be found in Additional file 1: Table S4. After applying the Benjamini-Hochberg correction, the differences in TRT\% were not significantly different.

The CNN resulted in a TRT\% of more than $10 \%$ in 3 out of 28 cases, while the conventional methods resulted in a TRT\% higher than $10 \%$ in 12 (MV2, SUV4, $41 \% \mathrm{SUV}_{\mathrm{MAX}}$ ) or 13 cases(MV3). The TF segmentation resulted in a TRT\% of more than $10 \%$ in only 8 cases.

TF, CNN and MV2 yielded similar ICCs (TF: 0.98, MV2: $0.97, \mathrm{CNN}$ : 0.99) indicating a very good repeatability. MV3, SUV4 and 41\% SUVMAX resulted in ICC of $0.73,0.81$ and 0.68 , respectively. The lesion size did not influence the repeatability of the segmentations.

\section{Summary of the results}

In summary, $\mathrm{CNN}$ and TF segmentation resulted in a better repeatability when compared with conventional approaches. Furthermore, both approaches resulted in a good accuracy when compared with the reference segmentations. The observed differences between the two AI-based methods were not significant for accuracy nor for repeatability. Therefore, our results suggest that both AI methods are good candidates for the segmentation of 

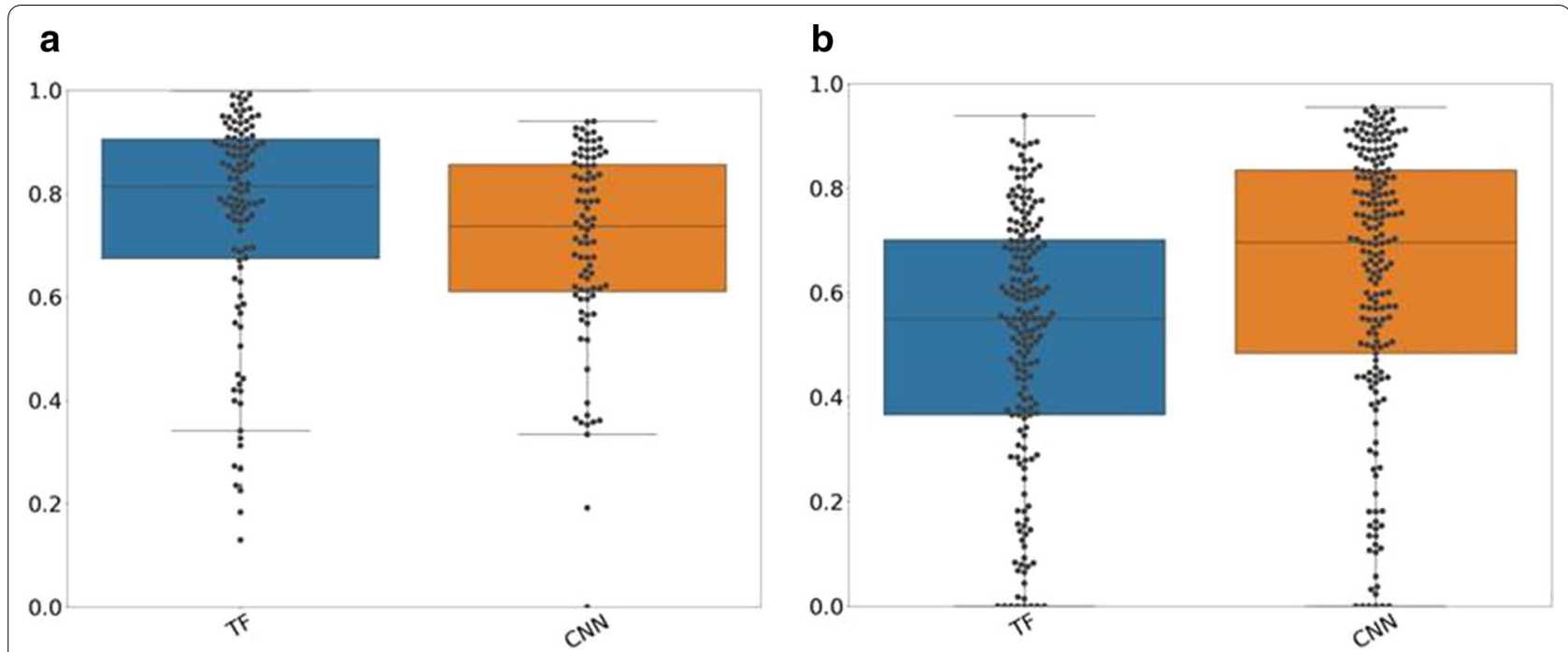

Fig. 5 Jaccard coefficient (JC) values dependent on lesion size: JC values for bigger (left figure) and smaller (right figure) lesions for both Al-based segmentation approaches

Table 2 Accuracy metrics for smaller and bigger lesions

\begin{tabular}{|c|c|c|c|c|c|c|}
\hline & $\begin{array}{l}\text { JC bigger } \\
\text { Median (25th/75th } \\
\text { quar) }\end{array}$ & $\begin{array}{l}\text { Volume ratio diff } \\
\text { bigger } \\
\text { Median (25th/75th } \\
\text { quar) }\end{array}$ & $\begin{array}{l}\text { Barycentric } \\
\text { distance } \\
\text { Median (25th/75th } \\
\text { quartile) }\end{array}$ & $\begin{array}{l}\text { JC } \\
\text { Median ( } 25 \text { th } / 75 \text { th } \\
\text { quar) smaller }\end{array}$ & $\begin{array}{l}\text { Volume ratio } \\
\text { smaller } \\
\text { Med (25th/75th } \\
\text { quar) }\end{array}$ & $\begin{array}{l}\text { Barycentric } \\
\text { distance } \\
\text { Median (25th/75th } \\
\text { quartile) }\end{array}$ \\
\hline TF & $0.82(0.64 / 0.89)$ & $0.91(0.81 / 1.13)$ & $0.61(0.4 / 1.3)$ & $0.56(0.39 / 0.68)$ & $0.91(0.55 / 0.95)$ & $0.76(0.46 / 1.8)$ \\
\hline CNN & $0.74(0.59 / 0.83)$ & $0.92(0.79 / 1.1)$ & $1.1(0.4 / 2.6)$ & $0.7(0.54 / 0.82)$ & $0.92(0.73 / 1.18)$ & $0.77(0.31 / 1.9)$ \\
\hline
\end{tabular}

NSCLC tumors in PET images and are more powerful than conventional approaches in terms of repeatability. However, use of these AI methods for other tumor types requires further validation and most likely additional (transfer or re-) training.

\section{Discussion}

In this paper, we evaluated two AI-based segmentation approaches in terms of repeatability and analyzed their accordance with a reference segmentation. Both approaches resulted in a good accuracy when compared with the reference segmentation used. The differences in performance between both AI approaches were small and statistically non-significant.

The segmentation of smaller lesions remains also for these two AI approaches a challenging task. One reason might be that with decreasing tumor size, small misclassifications have a higher impact on accuracy metrics as illustrated in Additional file 1: Table S5. Smaller lesions also typically show a lower tumor-tobackground ratio and are therefore more difficult to segment. This might be the reason that the CNN was not able to delineate some smaller lesions completely. Moreover, some metastasis are located close to other high-uptake regions (such as the kidney) and distinguishing tumor from normal uptake is in this case challenging for any segmentation algorithm and often requires manual correction. Especially for the $\mathrm{CNN}$, the different locations of the metastasis and therefore the differences in surrounding tissue yield a more challenging learning task than the segmentation of primary lung tumors alone.

In terms of accuracy and precision, the CNN trained and tested in this study was comparable with previous CNNs designed for the segmentation of primary tumors in PET images. An important difference between our methods and other published algorithm is that our approaches rely on the PET image information only and can therefore also be used when only a low-dose CT is acquired aside of the PET image $[14,16]$. Previous studies reported low segmentation performance when using the PET image for segmentation only $[16,18]$.

The CNN used in this paper is implemented with a relatively low number of features and layers when 


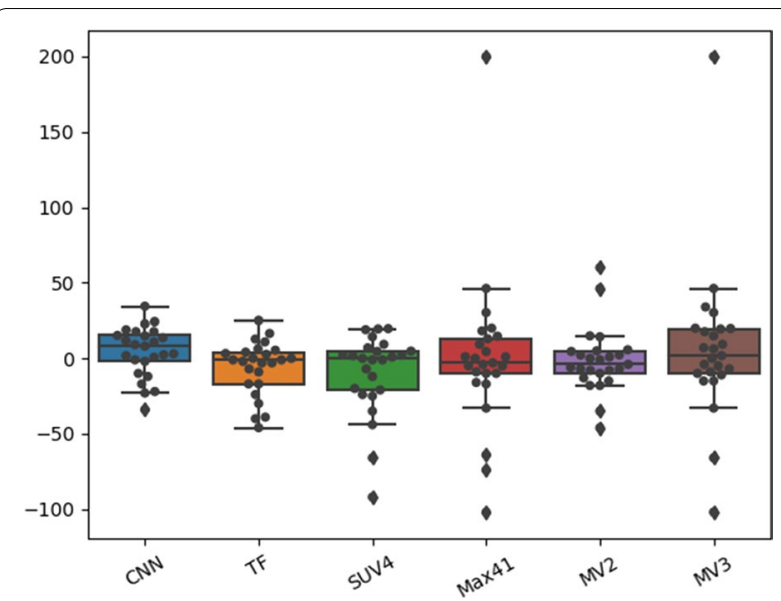

Fig. 6 Test-retest coefficient (TRT\%) for all segmentation approaches: If the TRT\% is close to 0 , the repeatability of the segmentations is excellent. Abbreviations of the segmentation algorithm: SUV4 standardized uptake value 4, 41\%SUV MAX $_{1}$ MV2 majority vote 2, MV3 majority vote 3, TF textural feature-based approach, CNN convolutional neural network

compared with the original U-Net or other CNNs designed for the segmentation of tumors in medical images $[15,23]$. Due to the relatively small dataset in the present study, we found that these combination of numbers of features and layers prevents the network from over-fitting while still yielding good results. A possible reason why the network performs well with a small number of features might be that in this study, the $\mathrm{CNN}$ is trained on tumor segmentation in a predefined bounding box and does not need to detect the tumor.

When the tumor was located close to other high uptake regions such as the heart or the kidneys, both segmentation approaches delineated this normal tissue high-uptake region as tumor. The standard automatic segmentation methods included in this study are mainly intensity driven. and they are therefore also not able to distinguish tumor from high-uptake regions when both are in close proximity. For these cases, it is likely that human interaction will always remain necessary, as mentioned previously [27]. However, in future studies, we will investigate if these segmentation approaches might also be used for lesion detection.

Also when compared with previous studies, the CNN and TF approaches outperformed other (semi-) automatic segmentation methods. Frings et al. reported a TRT\% repeatability coefficient of 44.4-71.1 for all lesions included in their analysis when using different thresholdbased segmentation approaches with background correction [28]. The AI-based segmentation methods yielded repeatability coefficients of $31.36(\mathrm{CNN})$ and 33.36 (TF), which are better than those reported by Frings et al. For images acquired under the same conditions as in our study (i.e., 60 min time between tracer injection and scan start and EARL-compliant reconstructions), Kolinger et al. found repeatability coefficients of 43 to 56 *, which are also higher than the ones of our AI-based segmentations [7]. However, Kolinger et al. reported lower repeatability coefficients for MV3 and 41\% SUV $\mathrm{MAX}$ segmentation approaches. The reason for this might be that Kolinger et al. compared the repeatability for the summed MATV of all lesions (TMATV), while we compared the repeatability of MATV for each lesion separately. A discrepancy in the segmentation of one lesion, especially if the lesion is small, has less impact on the repeatability of TMATV.

A disadvantage of AI-based segmentation approaches is the need for reliable training data. The lack of reasonable training data is one drawback making the clinical implementation of AI-based segmentation algorithms challenging. However, the MV2 approach used in this study was found to result in accurate and robust segmentations in a previous study [7]. Moreover, in our study, it also outperformed the conventional segmentation approaches in terms of repeatability without depending on training data. Especially for tasks where segmentation accuracy is important, such as radiotherapy planning, the MV2 is a good candidate for clinical use. Yet, regardless the method used, the final segmentation should always be supervised. In terms of repeatability, the CNN segmentation outperformed the MV2 approach and is the method of choice when segmentation repeatability is important, such as for longitudinal studies and/or for the evaluation of treatment response.

Another drawback of AI-based segmentation approaches is that they are trained for one specific task such as the segmentation of lung tumors or head and neck tumors. To apply the already trained algorithms to other (even similar tasks) requires a re-training of the algorithms. Therefore, both segmentation algorithms trained and validated in this study are likely not directly applicable to other cancer types such as head and neck cancer. Before using them for another cancer types, the algorithms need to be re-trained or at least undergo rigorous validation.

One limitation of this study is that the reference segmentations were delineated by one, yet experienced, observer while the consensus of three expert segmentations is considered as gold standard. To account for this, the segmentation was initiated with a semi-automated delineation method, an approach known to reduce observer variability. Of note, for the test-retest study, the same lesions were delineated by 5 observers in a previous study (7), and it was shown that even the consensus contour of these observers was less repeatable 
than those seen with any of the automated approaches. Finally, in our repeatability study, we included the AIbased approaches as well as several conventional methods and this repeatability study showed that our trained AI approaches provided very good results, even if the ground truth segmentations used during training of the AI methods would have been suboptimal.

Another limitation is the small dataset used for repeatability analysis. However, the collection of testretest scans is unfortunately limited due to the patient burden coming with consecutive scans of the same patients. Future studies, especially studies using data from different centers should confirm our findings.

\section{Conclusion}

In this paper, we compared the repeatability of AIbased segmentation algorithm with conventional segmentation approaches. Our results illustrate the advantage of AI-based segmentation approaches: Both approaches resulted in a good accuracy when compared with the reference segmentation and showed a high repeatability. Together with a majority vote approach (combining the results of four conventional segmentation approaches) the proposed AI-based segmentation methods were superior to the other segmentation algorithms included in this study in terms of repeatability. This study demonstrates that AI-based segmentations have not only the potential to accurately segment lesions but also result in more repeatable segmentations.

\section{Supplementary Information}

The online version contains supplementary material available at https://doi. org/10.1186/s13550-020-00744-9.

Additional file 1: Supplemental material.

\section{Abbreviations}

PET: Positron emission tomography: MATV: Metabolic active tumor volume; TMATV: Total MATV; TLG: Total lesion glycolysis; TF: Textural feature; CNN: Convolutional neural network; JC: Jaccard coefficient; TRT: Test-retest coefficient; ICC: Intraclass correlation coefficient; FDG: Fluorodeoxyglucose; CT: Computed tomography; Al: Artificial intelligence; NSCLC: Non-small cell lung cancer; SUV: Standardized uptake value; MV: Majority vote.

\section{Acknowledgements}

We would like to thank the Center for Information Technology of the University of Groningen for their support and for providing access to the Peregrine high performance computing cluster.

\section{Authors' contributions}

EP implemented the segmentation methods, analyzed the data, and wrote the manuscript. LM, GK, PA, MT, KV contributed to the patient inclusion, data acquisition, and manuscript revision. JdJ contributed to manuscript revision. OSH contributed to the patient inclusion, data acquisition, and manuscript revision. $\mathrm{RB}$ designed and managed the study, developed software for image processing, and wrote the manuscript. All authors read and approved the final manuscript.

\section{Funding}

This work is part of the research program STRaTeGy with Project Number 14929, which is (partly) financed by the Netherlands Organization for Scientific Research (NWO). This study was financed by the Dutch Cancer Society, POINTING Project, Grant 10034

\section{Availability of data and materials}

The datasets used and/or analyzed during the current study are available from the corresponding author on reasonable request.

\section{Ethics approval and consent to participate}

All procedures performed in studies involving human participants were in accordance with the ethical standards of the institutional and/or national research committee and with the 1964 Helsinki declaration and its later amendments or comparable ethical standards.

\section{Consent for publication}

Not applicable.

\section{Competing interests}

The authors declare that they have no competing interests.

\section{Author details}

${ }^{1}$ Department of Nuclear Medicine and Molecular Imaging, Medical Imaging Center, University of Groningen, University Medical Center Groningen, Groningen, The Netherlands. ${ }^{2}$ Faculty of Medicine and Life Sciences, Hasselt University, Agoralaan Building D, 3590 Diepenbeek, Belgium. ${ }^{3}$ Department of Nuclear Medicine, Ziekenhuis Oost Limburg, Schiepse Bos 6, 3600 Genk, Belgium. ${ }^{4}$ Department of Radiology and Nuclear Medicine, VU University Medical Center, Amsterdam, The Netherlands. ${ }^{5}$ Department of Respiratory Medicine, Ziekenhuis Oost Limburg, Schiepse Bos 6, 3600 Genk, Belgium. ${ }^{6}$ Department of Respiratory Medicine, AZ Vesalius Hospital, Hazelereik 51, 3700 Tongeren, Belgium. ${ }^{7}$ Institute for Materials Research (IMO) - Division Chemistry, Hasselt University, Agoralaan Building D, 3590 Diepenbeek, Belgium.

Received: 5 August 2020 Accepted: 10 December 2020 Published online: 06 January 2021

\section{References}

1. Volpi S, Ali JM, Tasker A, et al. The role of positron emission tomography in the diagnosis, staging and response assessment of non-small cell lung cancer. Ann Transl Med. 2018;6:95-95. https://doi.org/10.21037/ atm.2018.01.25.

2. Griffeth LK. Use of PET/CT scanning in cancer patients: technical and practical considerations. Proc (Bayl Univ Med Cent). 2005;18:321-30. https ://doi.org/10.1080/08998280.2005.11928089.

3. Hammerschmidt S, Wirtz H. Lung cancer. Dtsch Aerzteblatt Online. 2009. https://doi.org/10.3238/arztebl.2009.0809.

4. Vorwerk H, Beckmann G, Bremer M, et al. The delineation of target volumes for radiotherapy of lung cancer patients. Radiother Oncol. 2009:91:455-60. https://doi.org/10.1016/j.radonc.2009.03.014.

5. Johansson J, Alakurtti K, Joutsa J, et al. Comparison of manual and automatic techniques for substriatal segmentation in 11C-raclopride high-resolution PET studies. Nucl Med Commun. 2016;37:1074-87. https ://doi.org/10.1097/MNM.0000000000000559.

6. Hatt M, Lee JA, Schmidtlein CR, et al. Classification and evaluation strategies of auto-segmentation approaches for PET: report of AAPM task group No. 211. Med Phys. 2017;44:e1-42. https://doi.org/10.1002/ mp.12124.

7. Kolinger GD, Vállez García D, Kramer GM, et al. Repeatability of [18F]FDG PET/CT total metabolic active tumour volume and total tumour burden in NSCLC patients. EJNMMI Res. 2019;9:14. https://doi.org/10.1186/s1355 0-019-0481-1.

8. Schinagl DAX, Vogel WV, Hoffmann AL, et al. Comparison of five segmentation tools for 18F-fluoro-deoxy-glucose-positron emission 
tomography-based target volume definition in head and neck cancer. Int J Radiat Oncol. 2007;69:1282-9. https://doi.org/10.1016/j.jjrob p.2007.07.2333.

9. Jentzen W, Freudenberg L, Eising EG, et al. Segmentation of PET volumes by iterative image thresholding. J Nucl Med. 2007;48:108-14.

10. Nestle U, Kremp S, Schaefer-Schuler A, et al. Comparison of different methods for delineation of ${ }^{18} \mathrm{~F}$-FDG PET-positive tissue for target volume definition in radiotherapy of patients with non-Small cell lung cancer. J Nucl Med. 2005:46:1342-8.

11. Halt M, Le Rest CC, Turzo A, et al. A fuzzy locally adaptive Bayesian segmentation approach for volume determination in PET. IEEE Trans Med Imaging. 2009;28:881-93. https://doi.org/10.1109/TMI.2008.2012036.

12. Foster B, Bagci $U$, Mansoor A, et al. A review on segmentation of positron emission tomography images. Comput Biol Med. 2014;50:76-96. https:// doi.org/10.1016/j.compbiomed.2014.04.014.

13. Zhang $Y$, Oikonomou A, Wong A, et al. Radiomics-based prognosis analysis for non-small cell lung cancer. Sci Rep. 2017;7:46349. https://doi. org/10.1038/srep46349.

14. Zhong Z, Kim Y, Zhou L, et al. 3D fully convolutional networks for cosegmentation of tumors on PET-CT images. In: Proceedings -international symposium on biomedical imaging; 2018. p. 228-31. https://doi. org/https://doi.org/10.1109/ISBI.2018.8363561.

15. Blanc-Durand P, Van Der Gucht A, Schaefer N, et al. Automatic lesion detection and segmentation of 18F-FET PET in gliomas: a full 3D U-Net convolutional neural network study. PLOS ONE. 2018;13:1-11. https://doi. org/10.1371/journal.pone.0195798.

16. Yu H, Caldwell C, Mah K, Mozeg D. Coregistered FDG PET/CT-based textural characterization of head and neck cancer for radiation treatment planning. IEEE Trans Med Imaging. 2009;28:374-83. https://doi. org/10.1109/TMI.2008.2004425.

17. $\mathrm{Yu} \mathrm{H}$, Caldwell $\mathrm{C}$, Mah $\mathrm{K}$, et al. Automated radiation targeting in head-and-neck cancer using region-based texture analysis of PET and CT images. Int J Radiat Oncol Biol Phys. 2009;75:618-25. https://doi. org/10.1016/j.ijrobp.2009.04.043

18. Markel D, Caldwell C, Alasti H, et al. Automatic segmentation of lung carcinoma using 3D texture features in 18-FDG PET/CT. Int J Mol Imaging. 2013;2013:1-13. https://doi.org/10.1155/2013/980769.

19. Aide $N$, Lasnon $C$, Veit-Haibach $P$, et al. EANM/EARL harmonization strategies in PET quantification: from daily practice to multicentre oncological studies. Eur J Nucl Med Mol Imaging. 2017;44:17-31. https://doi. org/10.1007/s00259-017-3740-2.

20. Boellaard R, Delgado-Bolton R, Oyen WJG, et al. FDG PET/CT: EANM procedure guidelines for tumour imaging: version 2.0. Eur J Nucl Med Mol Imaging. 2015;42:328-54. https://doi.org/10.1007/s00259-014-2961-x.

21. Vanhove K, Mesotten L, Heylen M, et al. Prognostic value of total lesion glycolysis and metabolic active tumor volume in non-small cell lung cancer. Cancer Treat Res Commun. 2018;15:7-12. https://doi.org/10.1016/j. ctarc.2017.11.005.

22. van Baardwijk A, Bosmans G, Boersma L, et al. PET-CT-based autocontouring in non-small-cell lung cancer correlates with pathology and reduces interobserver variability in the delineation of the primary tumor and involved nodal volumes. Int J Radiat Oncol Biol Phys. 2007;68:771-8. https://doi.org/10.1016/j.ijrobp.2006.12.067

23. Ronneberger O, Fischer P, Brox T. U-Net: convolutional networks for biomedical image segmentation; 2015.

24. Van GJJM, Fedorov A, Parmar C, et al. Computational radiomics system to decode the radiographic phenotype. Cancer Res. 2017;77:104-8. https:// doi.org/10.1158/0008-5472.CAN-17-0339.

25. Pfaehler E, Mesotten L, Kramer G, et al. Textural feature based segmentation: a repeatable and accurate segmentation approach for tumors in PET images; 2020. pp. 3-14

26. Koo TK, Li MY. A guideline of selecting and reporting intraclass correlation coefficients for reliability research. J Chiropr Med. 2016;15:155-63. https:// doi.org/10.1016/j.jcm.2016.02.012

27. Hatt M, Laurent B, Ouahabi A, et al. The first MICCAI challenge on PET tumor segmentation. Med Image Anal. 2018:44:177-95. https://doi. org/10.1016/j.media.2017.12.007.

28. Frings $V$, de Langen AJ, Smit EF, et al. Repeatability of metabolically active volume measurements with 18F-FDG and 18F-FLT PET in non-small cell lung cancer. J Nucl Med. 2010;51:1870-7. https://doi.org/10.2967/jnume d.110.077255.

\section{Publisher's Note}

Springer Nature remains neutral with regard to jurisdictional claims in published maps and institutional affiliations.

\section{Submit your manuscript to a SpringerOpen ${ }^{\circ}$ journal and benefit from:}

- Convenient online submission

- Rigorous peer review

- Open access: articles freely available online

- High visibility within the field

Retaining the copyright to your article

Submit your next manuscript at springeropen.com 